\title{
A study of bacteriological and antibiotic susceptibility profile of urinary tract infection
}

\author{
Anusuya Devi D. ${ }^{1}$, Naik N. ${ }^{2}$, Krishnamurthy V. ${ }^{3}$ \\ ${ }^{1}$ Dr. Anusuya Devi D, Assistant Professor, ${ }^{2}$ Dr. Neelesh Naik, Associate Professor, ${ }^{3}$ Dr. Veena Krishnamurthy, Professor; \\ all authors are affiliated with Department of Microbiology, Sri Siddhartha Medical College and Hospital, Tumkur, \\ Karnataka, India.
}

Corresponding Author: Dr. Neelesh Naik, Associate Professor, Department of Microbiology, Sri Siddhartha Medical College and Hospital, Agalakote, B.H Road, Tumkur, Karnataka. Email id: drneelnk@gmail.com

\begin{abstract}
Introduction: Urinary tract infection (UTI) is one of the most common infection and is associated with significant morbidity in the community. Most of the UTI cases are treated empirically with broad-spectrum antibiotics which invariably results in the development of resistance. Aims and Objectives: The objective of this study was to determine the antibiotic susceptibility pattern of bacterial isolates causing UTI and to determine Extended spectrum beta Lactamase (ESBL) production in Gram negative isolates. Materials and Methods: A total of 724 urine samples were studied and bacteria identified by standard microbiological methods. Antibiotic sensitivity pattern was done by Kirby-Bauer disc diffusion method. Detection of ESBL was done as per Clinical and Laboratory Standards Institute (CLSI) guidelines. Results: Significant bacteriuria was detected in $238(32.8 \%)$ samples. The most common pathogens isolated were Escherichia coli 148 (58.9\%), Klebsiella pneumoniae 57 (22.7\%) and Staphylococcus aureus 18 (7.1\%) followed by Enterococcus spp 7 (2.7\%), Proteus mirabilis 6 (2.4\%), Citrobacter koseri 6 (2.4\%), Pseudomonas aeruginosa 5(2\%) and Staphylococcus saphrophyticus 4 (1.6\%). ESBL production was seen in Klebsiella pneumoniae 12 (21\%), followed by Escherichia coli 26 (17.5\%). Most of the Gram-negative bacteria were susceptible to meropenam, piperacillintazobactum and nitrofurantoin. Conclusion: This study reveals that many bacteria causing UTI are multidrug resistant pathogens. This suggests that regular monitoring and modification of empirical therapy and it's validation by culture report is required to prevent morbidity associated with this disease.
\end{abstract}

Keywords: Urinary tract infection, Extended spectrum beta lactamase, Drug resistance.

\section{Introduction}

Urinary tract infections (UTI) is the most common bacterial infections that lead to seek medical care. About 150 million people develop UTI each year globally [1]. UTI are also the most common hospitalacquired infections, accounting for as many as $40 \%$ of nosocomial infections [2]. The problem of UTI involve both males and females of all age groups including neonates. Malnutrition, low socio-economic status with poor hygiene, structural and functional abnormalities of urinary tract are few of the main predisposing factors causing UTI [3]. Escherichia coli, Klebsiella pneumoniae, Staphylococcus aureus, Coagulase negative staphylococci, Proteus mirabilis, Pseudomonas aeruginosa are the most common pathogenic microorganisms isolated in urine [4]. Most of the UTI cases are treated empirically with broad spectrum

Manuscript received: $8^{\text {th }}$ August 2018

Reviewed: $18^{\text {th }}$ August 2018

Author Corrected: $24^{\text {th }}$ August 2018

Accepted for Publication: $27^{\mathrm{th}}$ August 2018 antibiotics without the use of culture and sensitivity testing to guide therapy. Treatment becomes even more challenging in the presence of risk factors such as higher age, co-morbid conditions like diabetes mellitus, renal stones and immunosuppression [5]. Extensive and inappropriate use of antibiotics has invariably resulted in the development of antibiotic resistance which has become a major problem worldwide [6].

Extended spectrum beta-lactamase (ESBL) are the results of mutations in the ubiquitous class A TEM or SHV beta-lactamases. TEM-1 accounts for the majority of beta-lactamase-mediated resistance.These are mainly produced by Escherichia coli and Klebsiella [7].

ESBL producing bacteria show resistance not only to penicillins, cephalosporins and aztreonam but also to other classes of antibiotics such as aminoglycosides, cotrimoxazole, tetracycline and fluoroquinolones [8]. 
This increasing antimicrobial resistance complicates an uncomplicated UTI treatment by increasing patient morbidity, prolonged hospital stay, retreatment and use of broader spectrum of antibiotics.

Knowledge of the antimicrobial resistance pattern of common uropathogens according to local epidemiology is essential for providing clinically appropriate and costeffective therapy for UTI. Thus, this study was carried out to determine the prevalent uropathogens and antibiotic resistance patterns in our hospital.

\section{Materials and Methods}

Place of study: This study was carried out in the department of Microbiology at Sri Siddhartha Medical College, Hospital \& Research Centre, Tumkur, during the period of January 2016 to December 2016.

Type of study: Prospective study

Inclusion criteria: Clinically suspected cases of UTI

Exclusion criteria: Patients on antibiotics in prior week were excluded from the study.

Sample collection and method: A total of 724 consecutive, nonrepetitive urine samples were included in this study. A loopful $(0.001 \mathrm{ml})$ of well mixed uncentrifuged urine was inoculated onto blood agar, Mac Conkey's agar and cysteine-lactose electrolyte deficient (CLED) agar.

All plates were then incubated at $37^{\circ} \mathrm{C}$ for $24 \mathrm{hrs}$. Significant growth was considered if colony count $\geq 10^{5}$ colony forming unit $/ \mathrm{ml}(\mathrm{CFU} / \mathrm{ml})$ based on Kass concept.

All the isolates were identified biochemically by the standard microbiological methods [9, 10].

Antimicrobial susceptibility testing: This was done on Muller Hinton agar by Kirby Bauer disc diffusion method according to the CLSI guidelines (11).

\section{Original Research Article}

All Enterobacteriaceae members were tested against ampicillin/ sulbactum $(10 \mu \mathrm{g} / 10 \mu \mathrm{g})$, nitrofurantoin $(300 \mu \mathrm{g})$, amikacin $(30 \mu \mathrm{g}), \quad$ gentamicin $(10 \mu \mathrm{g})$, cefotaxime $(30 \mu \mathrm{g})$, ceftriaxone $(30 \mu \mathrm{g})$, ceftazidime $(30 \mu \mathrm{g})$, cotrimoxazole $(1.25 / 23.75 \mu \mathrm{g})$, ofloxacin $(10 \mu \mathrm{g})$, piperacillin- tazobactum $(100 / 10 \mu \mathrm{g})$ and meropenem $(10 \mu \mathrm{g})$.

Staphylococci were tested against ampicillin/ sulbactum $(10 \mu \mathrm{g} / 10 \mu \mathrm{g})$, amikacin $(30 \mu \mathrm{g})$, gentamicin $(10 \mu \mathrm{g})$, ceftriaxone $(30 \mu \mathrm{g})$, ciprofloxacin $(10 \mu \mathrm{g})$, nitrofurantoin $(300 \mu \mathrm{g})$, cotrimoxazole $(1.25 / 23.75 \mu \mathrm{g})$, vancomycin $(30 \mu \mathrm{g})$, linezolid $(30 \mu \mathrm{g})$ and cefoxitin $(30 \mu \mathrm{g})$.

Enterococcus spp were tested against amikacin $(30 \mu \mathrm{g})$, high level gentamicin $(120 \mu \mathrm{g})$ ceftriaxone $(30 \mu \mathrm{g})$ vancomycin $(30 \mu \mathrm{g})$, ciprofloxacin $(10 \mu \mathrm{g})$, nitrofurantoin $(300 \mu \mathrm{g})$ and linezolid $(30 \mu \mathrm{g})$.

Test for Detection of ESBL Production in Enterobacteriaceae: Isolates which were resistant to third generation cephalosporins were tested for ESBL production by combination disk method using cefotaxime $(30 \mu \mathrm{g})$, cefotaxime/ clavulanic acid (30 $\mu \mathrm{g} / 10 \mu \mathrm{g})$, and ceftazidime $(30 \mu \mathrm{g})$, ceftazidime/ clavulanic acid $(30 \mu \mathrm{g} / 10 \mu \mathrm{g})$.

Plates were incubated overnight at $37^{\circ} \mathrm{C}$. Zone of inhibition of $\geq 5 \mathrm{~mm}$ around cephalosporin + clavulanate compared to cephalosporin alone confirms ESBL production [11].

Test for detection of Methicillin resistance in Staphylococcus: The test was carried out on MullerHinton agar using a cefoxitin disc $(30 \mu \mathrm{g})$ and incubated at $35^{\circ} \mathrm{C}$ for $18-24 \mathrm{hrs}$.

An inhibition zone diameter of $\leq 21 \mathrm{~mm}$ was reported as methicillin resistant and a diameter of $\geq 22 \mathrm{~mm}$ was reported as methicillin sensitive strains [11].

\section{Results}

Out of 724 urine samples, significant bacteriuria was seen in $238(32.8 \%)$ samples which yielded 251 isolates.

The prevalence of UTI was higher in females (66.8\%) and prevalence among males was (33.2\%). $74(10.2 \%)$ patients had an insignificant colony count.

No growth was seen in $345(47.6 \%)$ specimens and mixed insignificant growth was seen in 67 (9.25\%) samples.

The most common isolates were Escherichia coli 148 (58.9\%), Klebsiella pneumoniae 57 (22.7\%), Staphylococcus aureus 18 (7.1\%) followed by Enterococcus spp 7 (2.7\%), Proteus mirabilis 6 (2.4\%), Citrobacter koseri 6 (2.4\%), Pseudomonas aeruginosa 5(2\%) and Staphylococcus saphrophyticus 4(1.6\%). 


\section{Original Research Article}

Table-1: Antibiotic sensitivity pattern of Gram negative organisms.

\begin{tabular}{|c|c|c|c|c|c|c|c|c|c|c|c|c|}
\hline $\begin{array}{l}\text { Sl. } \\
\text { No. }\end{array}$ & Isolates & $\begin{array}{c}\text { Amp/sul } \\
(\%)\end{array}$ & $\begin{array}{c}\mathbf{G} \\
(\%)\end{array}$ & $\begin{array}{l}\text { Ak } \\
(\%)\end{array}$ & $\begin{array}{l}\text { Of } \\
(\%)\end{array}$ & $\begin{array}{l}\text { Co } \\
(\%)\end{array}$ & $\begin{array}{l}\text { Nit } \\
(\%)\end{array}$ & $\begin{array}{l}\mathrm{Ca} \\
(\%)\end{array}$ & $\begin{array}{l}\mathrm{Ce} \\
(\%)\end{array}$ & $\begin{array}{l}\text { Ctr } \\
(\%)\end{array}$ & $\begin{array}{l}\text { Pt } \\
(\%)\end{array}$ & $\begin{array}{c}\text { M } \\
(\%)\end{array}$ \\
\hline 1 & $\begin{array}{c}\text { Escherichia coli } \\
\text { (148) }\end{array}$ & $\begin{array}{c}32 \\
(21.6) \\
\end{array}$ & $\begin{array}{c}98 \\
(66.2) \\
\end{array}$ & $\begin{array}{c}101 \\
(68.2)\end{array}$ & $\begin{array}{c}93 \\
(62.8) \\
\end{array}$ & $\begin{array}{r}77 \\
(52) \\
\end{array}$ & $\begin{array}{c}139 \\
(93.2)\end{array}$ & $\begin{array}{c}92 \\
(62.1) \\
\end{array}$ & $\begin{array}{c}88 \\
(59.4) \\
\end{array}$ & $\begin{array}{c}97 \\
(65.5) \\
\end{array}$ & $\begin{array}{c}140 \\
(94.5) \\
\end{array}$ & $\begin{array}{r}148 \\
(100) \\
\end{array}$ \\
\hline 2 & $\begin{array}{c}\text { Klebsiella } \\
\text { pneumonia }(57)\end{array}$ & $\begin{array}{c}12 \\
(21)\end{array}$ & $\begin{array}{c}34 \\
(59.6)\end{array}$ & $\begin{array}{c}36 \\
(63.1)\end{array}$ & $\begin{array}{c}31 \\
(54.3)\end{array}$ & $\begin{array}{c}26 \\
(45.6)\end{array}$ & $\begin{array}{c}53 \\
(92.9)\end{array}$ & $\begin{array}{c}34 \\
(59.6)\end{array}$ & $\begin{array}{c}32 \\
(56.1)\end{array}$ & $\begin{array}{c}37 \\
(64.9)\end{array}$ & $\begin{array}{c}52 \\
(91.2)\end{array}$ & $\begin{array}{c}57 \\
(100)\end{array}$ \\
\hline 3 & $\begin{array}{c}\text { Proteus mirabilis } \\
\text { (6) }\end{array}$ & $\begin{array}{c}1 \\
(16.6)\end{array}$ & $\begin{array}{c}2 \\
(33.3)\end{array}$ & $\begin{array}{c}2 \\
(33.3)\end{array}$ & $\begin{array}{c}2 \\
(33.3)\end{array}$ & $\begin{array}{c}3 \\
(50)\end{array}$ & $\begin{array}{c}4 \\
(66.6)\end{array}$ & $\begin{array}{c}6 \\
(100)\end{array}$ & $\begin{array}{c}6 \\
(100)\end{array}$ & $\begin{array}{c}6 \\
(100)\end{array}$ & $\begin{array}{c}6 \\
(100)\end{array}$ & $\begin{array}{c}6 \\
(100)\end{array}$ \\
\hline 4 & $\begin{array}{c}\text { Citrobacter koseri } \\
(6)\end{array}$ & $\begin{array}{c}2 \\
(33.3) \\
\end{array}$ & $\begin{array}{c}4 \\
(66.6) \\
\end{array}$ & $\begin{array}{c}4 \\
(66.6) \\
\end{array}$ & $\begin{array}{c}5 \\
(83.3) \\
\end{array}$ & $\begin{array}{c}3 \\
(50) \\
\end{array}$ & $\begin{array}{c}5 \\
(83.3) \\
\end{array}$ & $\begin{array}{c}5 \\
(83.3) \\
\end{array}$ & $\begin{array}{c}5 \\
(83.3) \\
\end{array}$ & $\begin{array}{c}6 \\
(100) \\
\end{array}$ & $\begin{array}{c}5 \\
(83.3) \\
\end{array}$ & $\begin{array}{c}6 \\
(100) \\
\end{array}$ \\
\hline 5 & $\begin{array}{l}\text { Pseudomonas } \\
\text { aeruginosa (5) }\end{array}$ & $\begin{array}{c}0 \\
(0)\end{array}$ & $\begin{array}{c}2 \\
(40)\end{array}$ & $\begin{array}{c}3 \\
(60)\end{array}$ & $\begin{array}{c}3 \\
(60)\end{array}$ & $\begin{array}{c}1 \\
(20)\end{array}$ & $\begin{array}{c}2 \\
(40)\end{array}$ & $\begin{array}{c}3 \\
(60)\end{array}$ & $\begin{array}{c}2 \\
(40)\end{array}$ & $\begin{array}{c}2 \\
(40)\end{array}$ & $\begin{array}{c}4 \\
(80)\end{array}$ & $\begin{array}{c}5 \\
(100)\end{array}$ \\
\hline & Total (222) & 47 & 140 & 146 & 134 & 110 & 203 & 140 & 133 & 148 & 207 & 222 \\
\hline
\end{tabular}

The predominant isolate Escherichia coli showed maximum sensitivity towards meropenem (100\%), piperacillintazobactum (94.5\%), nitrofurantoin (93.2\%) and they were least sensitive towards ampicillin/ sulbactum (21.6\%), ofloxacin $(37.5 \%)$ and cotrimoxazole (52\%). Klebsiella pneumoniae 57 (23.17\%) was the second most common isolated organism and it was most sensitivite to meropenem (100\%), nitrofurantoin (92.9\%) and piperacillin-tazobactum (91.2\%) and least sensitive to ampicillin/sulbactum (21\%), cotrimoxazole (45.6\%) and ofloxacin (54.3\%) (Table-1).

Table-2: Antibiotic sensitivity pattern of Escherichia coli.

\begin{tabular}{|c|c|c|c|c|c|c|c|c|c|c|c|c|}
\hline $\begin{array}{l}\text { Sl. } \\
\text { No. }\end{array}$ & $\begin{array}{c}\text { ESBL/ } \\
\text { Non-ESBL }\end{array}$ & $\begin{array}{c}\text { Amp/sul } \\
(\%)\end{array}$ & $\begin{array}{c}G \\
(\%)\end{array}$ & $\begin{array}{l}\text { Ak } \\
(\%)\end{array}$ & $\begin{array}{l}\text { Of } \\
(\%)\end{array}$ & $\begin{array}{c}\text { Co } \\
(\%)\end{array}$ & $\begin{array}{l}\text { Nit } \\
(\%)\end{array}$ & $\begin{array}{l}\mathrm{Ca} \\
(\%)\end{array}$ & $\begin{array}{c}\mathrm{Ce} \\
(\%)\end{array}$ & $\begin{array}{l}\mathrm{Ctr} \\
(\%)\end{array}$ & $\begin{array}{c}\mathbf{P t} \\
(\%)\end{array}$ & $\begin{array}{c}\mathrm{M} \\
(\%)\end{array}$ \\
\hline 1 & \begin{tabular}{|c|} 
ESBL \\
Escherichia coli-(26)
\end{tabular} & $\begin{array}{c}0 \\
(0)\end{array}$ & $\begin{array}{c}12 \\
(46.1)\end{array}$ & $\begin{array}{c}10 \\
(38.4)\end{array}$ & $\begin{array}{c}12 \\
(46.1)\end{array}$ & $\begin{array}{c}11 \\
(42.3)\end{array}$ & $\begin{array}{c}22 \\
(84.6)\end{array}$ & $\begin{array}{c}0 \\
(0)\end{array}$ & $\begin{array}{c}0 \\
(0)\end{array}$ & $\begin{array}{c}0 \\
(0)\end{array}$ & $\begin{array}{c}22 \\
(84.6)\end{array}$ & $\begin{array}{c}26 \\
(100)\end{array}$ \\
\hline 2 & \begin{tabular}{|c} 
Non ESBL \\
Escherichia coli $(122)$
\end{tabular} & $\begin{array}{c}32 \\
(26.2)\end{array}$ & $\begin{array}{c}86 \\
(70.4)\end{array}$ & $\begin{array}{c}91 \\
(74.5)\end{array}$ & $\begin{array}{c}81 \\
(66.3)\end{array}$ & $\begin{array}{c}66 \\
(54)\end{array}$ & $\begin{array}{c}117 \\
(95.9)\end{array}$ & $\begin{array}{c}92 \\
(75.4)\end{array}$ & $\begin{array}{c}88 \\
(72.1)\end{array}$ & $\begin{array}{c}97 \\
(79.5)\end{array}$ & $\begin{array}{c}118 \\
(96.7)\end{array}$ & $\begin{array}{c}122 \\
(100)\end{array}$ \\
\hline & Total 148 & 32 & 98 & 101 & 93 & 77 & 139 & 92 & 88 & 97 & 140 & 148 \\
\hline
\end{tabular}

Table-3: Antibiotic sensitivity pattern of Klebsiella pneumoniae.

\begin{tabular}{|c|c|c|c|c|c|c|c|c|c|c|c|c|}
\hline $\begin{array}{l}\text { Sl. } \\
\text { No. }\end{array}$ & $\begin{array}{c}\text { ESBL/ } \\
\text { Non-ESBL }\end{array}$ & $\begin{array}{c}\text { Amp/sul } \\
(\%)\end{array}$ & $\begin{array}{c}\mathbf{G} \\
(\%)\end{array}$ & $\begin{array}{c}\text { Ak } \\
(\%)\end{array}$ & $\begin{array}{c}\text { Of } \\
(\%)\end{array}$ & $\begin{array}{c}\text { Co } \\
(\%)\end{array}$ & $\begin{array}{l}\text { Nit } \\
(\%)\end{array}$ & $\begin{array}{l}\mathrm{Ca} \\
(\%)\end{array}$ & $\begin{array}{l}\mathrm{Ce} \\
(\%)\end{array}$ & $\begin{array}{l}\text { Ctr } \\
(\%)\end{array}$ & $\begin{array}{l}\text { Pt } \\
(\%)\end{array}$ & $\begin{array}{c}\text { M } \\
(\%)\end{array}$ \\
\hline 1 & $\begin{array}{l}\text { ESBL Klebsiella } \\
\text { pneumoniae (12) }\end{array}$ & $\begin{array}{c}0 \\
(0)\end{array}$ & $\begin{array}{c}4 \\
(33.3)\end{array}$ & $\begin{array}{c}4 \\
(33.3)\end{array}$ & $\begin{array}{c}3 \\
(25)\end{array}$ & $\begin{array}{c}0 \\
(0)\end{array}$ & $\begin{array}{c}10 \\
(83.3)\end{array}$ & $\begin{array}{c}0 \\
(0)\end{array}$ & $\begin{array}{c}0 \\
(0)\end{array}$ & $\begin{array}{c}0 \\
(0)\end{array}$ & $\begin{array}{c}10 \\
(83.3)\end{array}$ & $\begin{array}{c}12 \\
(100)\end{array}$ \\
\hline 2 & $\begin{array}{c}\text { Non ESBL } \\
\text { Klebsiella } \\
\text { pneumoniae (45) }\end{array}$ & $\begin{array}{c}12 \\
(26.6)\end{array}$ & $\begin{array}{c}30 \\
(66.6)\end{array}$ & $\begin{array}{c}32 \\
(71.1)\end{array}$ & $\begin{array}{c}28 \\
(62.2)\end{array}$ & $\begin{array}{c}26 \\
(57.7)\end{array}$ & $\begin{array}{c}43 \\
(95.5)\end{array}$ & $\begin{array}{c}34 \\
(75.5)\end{array}$ & $\begin{array}{c}32 \\
(71.1)\end{array}$ & $\begin{array}{c}37 \\
(82.2)\end{array}$ & $\begin{array}{c}42 \\
(93.3)\end{array}$ & $\begin{array}{c}45 \\
(100)\end{array}$ \\
\hline & Total 57 & 12 & 34 & 36 & 31 & 26 & 53 & 34 & 32 & 37 & 52 & 57 \\
\hline
\end{tabular}

Amp/sul-Ampicillin/ sulbactum, G-Gentamicin, Ak-Amikacin, Of-Ofloxacin, Co-Cotrimoxazole, Nit-nitrofurantoin, CaCeftazidime, Ce-Cefotaxime, Ctr-ceftriaxone, Pt-Piperacillin/tazobactam, M-meropenem,

Among the 217 Enterobacteriaceae isolates, 64 (29.4\%) were showing multidrug resistance (MDR). Among these 64 MDR isolates, $38(59 \%)$ were ESBL producers. Highest prevalence of ESBL production was seen in Klebsiella pneumoniae 12 (21\%), followed by Escherichia coli 26 (17.5\%). The ESBL producing strains showed maximum sensitivity towards meropenem, nitrofurantoin and piperacillin-tazobactum and $100 \%$ resistance towards third generation cephalosporins and ampicillin/ sulbactum (Table-2, 3). 


\section{Original Research Article}

Table- 4: Antibiotic sensitivity pattern of Gram positive organisms.

\begin{tabular}{|c|c|c|c|c|c|c|c|c|c|c|c|}
\hline $\begin{array}{l}\text { SI. } \\
\text { No. }\end{array}$ & Organisms & $\begin{array}{c}\text { Amp/sul } \\
(\%)\end{array}$ & $\begin{array}{c}\text { G/HLG } \\
(\%)\end{array}$ & $\begin{array}{c}\mathrm{Ak} \\
(\%)\end{array}$ & $\begin{array}{l}\text { Ctr } \\
(\%)\end{array}$ & $\begin{array}{l}\text { Cf } \\
(\%)\end{array}$ & $\begin{array}{c}\text { Co } \\
(\%)\end{array}$ & $\begin{array}{l}\text { Nit } \\
(\%)\end{array}$ & $\begin{array}{c}\mathrm{Lz} \\
(\%)\end{array}$ & $\begin{array}{c}\text { Cn } \\
(\%)\end{array}$ & $\begin{array}{c}\mathrm{Va} \\
(\%)\end{array}$ \\
\hline 1 & $\begin{array}{c}\text { Staphylococcus } \\
\text { aureus (18) }\end{array}$ & $\begin{array}{c}2 \\
(11.1)\end{array}$ & $\begin{array}{c}13 \\
(72.2)\end{array}$ & $\begin{array}{c}12 \\
(66.6)\end{array}$ & $\begin{array}{c}14 \\
(77.7)\end{array}$ & $\begin{array}{c}6 \\
(33.3)\end{array}$ & $\begin{array}{c}8 \\
(44.4)\end{array}$ & $\begin{array}{c}16 \\
(88.8)\end{array}$ & $\begin{array}{c}18 \\
(100)\end{array}$ & $\begin{array}{c}16 \\
(88.8)\end{array}$ & $\begin{array}{c}18 \\
(100)\end{array}$ \\
\hline 2 & $\begin{array}{l}\text { Enterococcus } \\
\text { species }(7)\end{array}$ & $\begin{array}{c}0 \\
(0)\end{array}$ & $\begin{array}{c}4 \\
(57)\end{array}$ & $\begin{array}{c}3 \\
(42)\end{array}$ & $\begin{array}{c}2 \\
(28)\end{array}$ & $\begin{array}{c}3 \\
(42)\end{array}$ & - & $\begin{array}{c}4 \\
(57)\end{array}$ & $\begin{array}{c}7 \\
(100)\end{array}$ & - & $\begin{array}{c}7 \\
(100)\end{array}$ \\
\hline 3 & $\begin{array}{c}\text { Staphylococcus } \\
\text { saphrophyticus (4) }\end{array}$ & $\begin{array}{c}0 \\
(0)\end{array}$ & $\begin{array}{c}2 \\
(50)\end{array}$ & $\begin{array}{c}2 \\
(50)\end{array}$ & $\begin{array}{c}2 \\
(50)\end{array}$ & $\begin{array}{c}3 \\
(60)\end{array}$ & $\begin{array}{c}2 \\
(50)\end{array}$ & $\begin{array}{c}4 \\
(100)\end{array}$ & $\begin{array}{c}4 \\
(100)\end{array}$ & $\begin{array}{c}4 \\
(100)\end{array}$ & $\begin{array}{c}4 \\
(100)\end{array}$ \\
\hline & Total-29 & 2 & 19 & 17 & 18 & 12 & 10 & 24 & 29 & 20 & 29 \\
\hline
\end{tabular}

Amp/sul-Ampicillin/sulbactum, G-Gentamicin, HLG-high level Gentamicin, Ak-Amikacin, Ctr-ceftriaxone, CfCiprofloxacin, Co-Cotrimoxazole, Nit-nitrofurantoin, Lz-linezolid, Cn-cefoxitin, Va-vancomycin.

All the isolates of Staphylococcus aureus were sensitive to vancomycin and linezolid (Table-4). Among the Staphylococcus aureus, $2(11.1 \%$ ) were found to be MRSA by disc diffusion test. 50\% of MRSA strains were sensitive to amikacin, gentamicin, ciprofloxacin and nitrofurantoin. They were $100 \%$ resistant to ampicillin/sulbactum, ceftriaxone and cotrimoxazole.

\section{Discussion}

For the appropriate empirical therapy of UTI, knowledge about present trends of the uropathogens and their susceptibility to various antibiotics is essential because studies have shown changing trends of susceptibility pattern from different places over a period of time $[12,13]$. Our study showed a high prevalence of UTI in females $(66.8 \%)$ than in males $(33.2 \%)$ which correlates with other findings done by Orrett et al, Sood et al $[14,15]$. UTI is more common in females because of shorter urethra and urethra is more proximal to anus so that coliforms enter and colonize urethra $[12,15]$.

Out of 724 urine samples, $238(32.8 \%)$ were found to be culture positive which yielded 251 isolates. Escherichia coli 148 (58.9\%) was the predominant uropathogen which is in concordance with the other studies $[2,16]$. Enterobacteriaceae have several factors responsible for their attachment to the uroepithelium. The Gram negative bacteria colonize the uroepithelium mucosa with adhesions, pili, fimbriae and P1 blood group phenotype [12, 17]. Klebsiella pneumoniae was the second most common organism isolated. Others have found an increase in Klebsiella pneumoniae causing UTI [18]. This increased trend may be due to increased colonization of multidrug resistant Klebsiella pneumoniae in hospital setup $[15,19]$.

The Gram-negative bacteria were showing maximum sensitivity towards meropenem and piperacillintazobactam followed by nitrofurantoin (91.4\%), amikacin (65.7\%) and gentamicin (63\%). According to Kaushik et al, the Gram-negative bacteria showed maximum sensitivity to nitrofurantoin (95.5\%), amikacin (75.5\%) and gentamicin (65.5\%) [2].The least sensitive antibiotic among the Gram-negative bacteria in our study was ampicillin/ sulbactum (21.1\%) followed by cotrimoxazole (49.5\%). In a study done by Sundaramurthy et al, after beta lactum antibiotics, fluoroquinolones were the least effective drugs followed by cotrimoxazole [20].

We observed $17.5 \%$ of the Escherichia coli and $21 \%$ of Klebsiella pneumoniae to be ESBL producers. In our study, both cefotaxime-clavulanic acid and ceftazidimeclavulanic acid identified ESBL producers equally, whereas in other studies cefotaxime-clavulanic acid identified more number of ESBL producers compared to ceftazidime-clavulanic acid $[19,21,22]$. ESBL strains apart from being resistant to third generation cephalosporins also showed more than $50 \%$ resistance to ampicillin/sulbactum, amikacin, gentamcin, ofloxacin and cotrimoxazole.

The important risk factors associated with ESBL producing organisms are prolonged hospital stay, long term usage of antibiotics, severe illness and catheterisation [23]. ESBL producing organisms do not respond to the empirical therapy of cephalosporins which leads to increased risk of morbidity and mortality and also increase in the cost of treatment [20]. Carbapenams, nitrofurantoin, piperacillin-tazobactum showed potent antibacterial activity against ESBL producing isolates which was similar to the results of other studies $[24,25]$. 


\section{Original Research Article}

Staphylococcus aureus $18(7.3 \%)$ was the most common Gram-positive bacteria followed by Enterococcus 7 (2.7\%), and Staphylococcus saphrophyticus 4 (1.6\%). 2 strains were of MRSA. Gram positive bacteria causing UTI is usually less compared to the Gram-negative bacteria but has gained significance due to the emergence of MRSA [26].

Some studies have isolated Enterococcus species as the commonest Gram-positive bacteria causing UTI followed by CONS $[27,28]$. The prevalence of different bacteria and their antibiotic resistance vary not only from place to place but also from institute to institute and this can be due to different health care settings, different antibiotic protocols and study population.

\section{Conclusion}

Knowledge of the uropathogens and their antimicrobial susceptibility pattern in an area is essential for providing effective therapy and control of UTI. Empirical therapy should be validated by culture report to prevent morbidity associated with the disease. To limit the spread of ESBL producing isolates, ESBL detection should be included in the routine antibiotic sensitivity testing. Continued surveillance, appropriate use of antibiotics and implementation of strict infection control measures are recommended to decrease ESBL production.

Importance of this study: The most common organisms causing UTI change from place to place and also their antibiotic sensitivity pattern. Hence, it was important to conduct this study in our hospital. We found that Escherichia coli was the most common organism causing UTI followed by Klebsiella pneumoniae. The Gram negative isolates were most sensitive to meropenem followed by piperacillintazobactam and nitrofurantoin, and all the Gram positive bacteria were sensitive to vancomycin, linezolid followed by nitrofurantoin. The antibiotic sensitivity pattern of the isolates will help in guiding therapy in our hospital.

Author contributions: First author has contributed in the concept and design of work, literature search, data acquisition, data analysis, manuscript preparation and manuscript review. Second author is the corresponding author and has contributed in the concept, design, literature search, data acquisition, data analysis, manuscript preparation and manuscript review. Third author has contributed in the data acquisition, manuscript editing and manuscript review.

Funding: Nil, Conflict of interest: None initiated Permission from IRB: Yes

\section{References}

1. Mokta JK, Verma S, Singh D, Kanga A. Bacterial etiology and antibiotic susceptibility pattern of urinary tract infection in sub-Himalayan region of India- a retrospective study of clinical isolates. National Journal of Medical and Allied Sciences 2015;4(1):38-45.

2. Kaushik C, Gangadhar NK, Subrahmanya BK, Kotigadde S. Anti-biogram pattern of uro-pathogens isolated from patients in a Tertiary Care Hospital in Karnataka, India. Indian J Microbiol Res 2018;5(1): 24-30.

3. Niranjan V, Malini A. Antimicrobial resistance pattern in Escherichia coli causing urinary tract infection among inpatients. Indian J Med Res 2014;139 (6): 945-8.

4. Renuart AJ, Goldfarb DM, Mokomane M, Tawanana EO, Narasimhamurthy M, Steenhoff AP. (2013). Microbiology of Urinary Tract Infections in Gaborone, Botswana. PLOS ONE. 4;8(3):e57776.

5. Arias CA, Murray BE. Antibiotic-resistant bugs in the 21st century--a clinical super-challenge. N Engl J Med 2009;29(5):439-43.

6. Aruna K, Mobashshera T. Prevalence of extended spectrum beta-lactamase production among uropathogens in south Mumbai and its antibiogram pattern. EXCLI Journal 2012;11(7):363-72.

7. Rupp ME, Fey PD. Extended spectrum $\beta$-lactamase (ESBL)- producing enterobacteriaceae: Staphylococcus saphrophyticus. Considerations for diagnosis, prevention and drug treatment. Drugs J 2003; 63 (4) : $353-65$.

8. Colodner R. Extended-spectrum beta-lactamases: a challenge for clinical microbiologists and infection control specialists. Am J Infect Control 2005;33(2): 104-7.

9. Deshmukh DG, Damle AS, Bajaj JK, Bhakre JB, Patwardhan NS. Metallo-ß-lactamase-producing clinical isolates from the patients of a tertiary care hospital. J Lab Physicians 2011;3:93-7.

10. Manjunath GN, Prakash R, Annam V, Shetty K. Changing trends in the spectrum of antimicrobial drug resistance pattern of uropathogens isolated from hospitals and community patients with urinary tract infections in Tumkur and Bangalore. Int J Biol Med Res 2011; 2(2):504-7. 


\section{Original Research Article}

11.Clinical and Laboratory Standards Institute. Performance standards for antimicrobial susceptibility testing; (2017).27 $7^{\text {th }}$ informational supplement, Wayne, PA, USA. 2017;32(3) M100-S27.

12. Magale Hi, Kassim Ia, Odera Sa, Omolo Mj, Jaoko Wg, Jolly Pe. Antibiotic Susceptibility Of Organisms Causing Urinary Tract Infection In Patients Presenting At Kenyatta National Hospital, Nairobi. East Afr Med J 2015;92(7):333-7.

13. Das RN, Chandrashekhar TS, Joshi HS, Gurung M, Shrestha N, Shivananda PG. Frequency and susceptibility profile of pathogens causing urinary tract infections at a tertiary care hospital in western Nepal. Singapore Med J 2006;47(4):281-5.

14. Gonzalez CM, Schaeffer AJ. Treatment of urinary tract infection: what's old, what's new, and what works. World J Urol 2001;17(6):372-82.

15. Orrett F A. Urinary tract infections in general practice in a rural community in South Trinidad. Saudi Med Journal 2001;22:537-40.

16. Sood S, Gupta R. Antibiotic resistance pattern of community acquired uropathogens at a tertiary care hospital in Jaipur, Rajasthan. Indian $\mathbf{J}$ of Communi Medi 2012;37:39-44.

17. Gupta N, Kundra S, Sharma A, GautamV, Arora DR. Antimicrobial susceptibility of uropathogens in India. J Infect Dis Antimicrob Agents 2007;24:13-8.

18. Prakash D, Saxena RS. Distribution and Antimicrobial Susceptibility Pattern of Bacterial Pathogens Causing Urinary Tract Infection in Urban Community of Meerut City, India. ISRN Microbiology 2013; 6:56-78.

19. Poudyal S, Bhatta DR, Shakya G, Upadhyaya B, Dumre SP, Buda G, et al. Extended Spectrum betalactamase producing multidrug resistant clinical bacterial isolates at National Public Health Laboratory, Nepal. Nepal Med Coll J 2011;13(1):34-8.

20. Sundaramurthy R, Tiruvanamalai R, Sivaraman ST, Arunagiri R, Charles J. Study on clinico microbiological profile and antibiotic susceptibility pattern of urinary tract pathogens with Special reference to susceptibility of Escherichia coli to fosfomycin. Indian J of Microbiol Res 2018;5(2):258-65

21. Nepal K, Pant ND, Neupane B, Belbase A, Baidhya R, Shrestha RK, et al. Extended spectrum beta-lactamase and metallo beta-lactamase production among Escherichia coli and Klebsiella pneumoniae isolated from different clinical samples in a tertiary care hospital in Kathmandu, Nepal . Ann Clin Microbiol Antimicrob 2017;16:62:1-7.

22. Yadav KK, Adhikari N, Khadka R, Pant AD, Shah B. Multidrug resistant Enterobacteriaceae and extended spectrum $\beta$-lactamase producing Escherichia coli: a cross-sectional study in National Kidney Center, Nepal. Antimicrob Resist Infect Control 2015;4:42.

23. Pilli R, Kapaganty VC. Study of extended spectrum beta lactamase producing uropathogens and their antibiotic susceptibility pattern. Indian $\mathrm{J}$ of Microbiol Res 2018;5(2):280-83.

24. Eshwarappa M, Dosegowda R, Aprameya IV, Khan MW, Kumar PS, Kempegowda P. Clinico-microbiological profile of urinary tract infection in South India. Indian J Nephrol 2011;21(1):30-3.

25. Kader AA, Kumar AK. Prevalence of extended spectrum beta-lactamase among multidrug resistant gram-negative isolates from a general hospital in Saudi Arabia. Saudi Med J 2004;25:570-4.

26. Shashwati N, Kiran T, Dhanvijay AG. Study of extended spectrum $\beta$-lactamase producing Enterobaceriaceae and antibiotic co-resistance in a tertiary care teaching hospital. J Nat Sci Biol Med 2014; 5:30-5.

27. Naik TB, Lavanya J, Upadhya A, Mani V. Gram positive uropathogens and their antibiogram: Data analysis at a tertiary care hospital in Karnataka. Indian J Microbiol Res 2018;5(1):71-5.

28. Bajpai T, Pandey M, Varma M, Bhatambare GS. Prevalence of extended spectrum beta-lactamase producing uropathogens and their antibiotic resistance profile in patients visiting a tertiary care hospital in central India: Implications on empiric therapy. Indian J Pathol Microbiol 2014;57(3):407-12.

\section{How to cite this article?}

Anusuya Devi D, Naik N, Krishnamurthy V. A study of bacteriological and antibiotic susceptibility profile of urinary tract infection. Trop J Path Micro 2018;4(4):324-329.doi:10. 17511/ jopm. 2018.i4.05 